\title{
SINGLE BUNCH COLLECTIVE EFFECTS IN PLS STORAGE RING
}

\author{
C. Kim*, Yujong Kim, K. H. Kim, J. Y. Huang, M. H. Cho, W. Namkung, and I. S. Ko \\ Pohang Accelerator Laboratory, POSTECH, Pohang 790-784, Korea
}

\section{Abstract}

The single bunch collective effects such as the bunch lengthening due to the potential well distortion and the microwave instability which also increases the energy spread, the transverse head-tail instability, and the transverse strong head-tail instability are investigated in the Pohang Light Source storage ring. The threshold current and the Chao-Gareyte scaling factor of the bunch lengthening, the longitudinal broadband impedance, and the transverse impedance have been measured and compared with the ZAP code results.

\section{INTRODUCTION}

In the storage ring, circulating electrons within a bunch will interact with the various discontinuous surroundings. Since the electron beams induce the electromagnetic wakefields on the wall of the surroundings via the the interaction, electron motions in its own bunch or the following bunches will be disturbed. Under some conditions, the disturbance of electron motions can be accumulated and generate the strong collective effects which invoke the loss of some or all beams. There are two kinds of wakefield in the time domain: one is the short range wakefield which is also called as the broadband impedance in the frequency domain, and the other is the long range wakefield which is also called as the narrowband impedance in the frequency domain. The short range wakefield or the broadband impedance is generated mainly due to the discontinuous vacuum components such as the steps, the bellows, and the BPM chambers as well as the RF cavities. Since the filling time or the duration time of the short range wakefield is short, it generates the single bunch collective effects such as the bunch lengthening due to the potential well distortion and the microwave instability which also increases the energy spread, the transverse head-tail instability, and the transverse strong head-tail instability. On the contrary, the long range wakefield is generated by mainly the RF cavities whose the duration time is long due to the high quality factor. Since the long range wakefield can disturb the election motions of the following bunches or its own bunch, it generates the longitudinal or transverse coupled bunch mode instabilities. In this paper, we have investigated the single bunch collective effects by measuring the bunch length and the energy spread of the single bunch and compared their results with those of the ZAP and the MOSES codes in the Pohang Light Source (PLS) storage ring [1], [2].

\footnotetext{
*chbkim@POSTECH.edu
}

\section{LONGITUDINAL EFFECTS}

\subsection{Potential Well Distortion}

The single bunch length of the electron storage ring is changed due to the potential well distortion and the microwave instability which also increases the energy spread. The space charge of circulating electron beams induces the electromagnetic fields which change the RF voltage or the potential well seen by the electrons. Therefore, the voltage change will generate the incoherent synchrotron oscillation frequency shift which will be also appeared as the change in the equilibrium bunch length. We call this phenomenon as the potential well distortion which still exist at the very low current. Since only the quantum fluctuations due to the synchrotron radiation can affect the energy spread of the radiating electron beams, and the energy spread is independent of the RF voltage, the potential well distortion does not generate any change in the energy spread. The relation between the single bunch length and the beam current due to the potential well distortion is given by

$$
\left(\frac{\sigma_{l}}{\sigma_{l o}}\right)^{3}-\left(\frac{\sigma_{l}}{\sigma_{l o}}\right)-\frac{\alpha I_{b} \operatorname{Im}\left\{Z_{\|} / n\right\}_{e f f}}{\sqrt{2 \pi}(E / e) \nu_{s o}^{2}}\left(\frac{R}{\sigma_{l o}}\right)^{3}=0,
$$

where $\sigma$ the distorted bunch length due to the potential well distortion, $\alpha$ is the momentum compaction factor, $I_{b}$ is the average beam current, $E$ is the beam energy, $e$ is the electron charge, $\nu_{s}$ is the synchrotron tune, $\operatorname{Im}\left\{Z_{\|} / n\right\}_{\text {eff }}$ is the effective longitudinal impedance, $R$ is the average radius of the storage ring, and the subscript zero means undistorted value which the potential well distortion is not considered [1], [3]. When the $\operatorname{Im}\left\{Z_{\|} / n\right\}_{\text {eff }}$ is negative or capacitive (positive or inductive), the bunch shortening (lengthening) will be generated due to the potential well distortion.

\subsection{Microwave Instability}

When the peak current of a single bunch higher than a threshold current, the different azimuthal modes of the bunch are coupled by the impedance of the beam surroundings. In this case, the energy spread and the bunch lengthening are generated until the peak current is reduced to the threshold peak beam current. We call this instability as the microwave instability or the turbulent bunch lengthening. The threshold peak beam current of the microwave instability is given by

$$
I_{p k}=\frac{\sqrt{2 \pi} R}{\sigma_{l}} I_{b}=\frac{2 \pi \alpha(E / e) \delta^{2}}{\left|Z_{\|} / n\right|},
$$

where $I_{p k}$ is the threshold peak current, $\sigma_{l}$ is the rms bunch length, $\delta$ is the rms fractional energy spread, $\left|Z_{\|} / n\right|$ is the 
longitudinal broadband impedance, others are the same as those of Eq. (1). Equation (2) is called the Keil-Schnell criterion of the microwave instability which is based on the coasting beam [1]. The bunch length above the threshold beam current follows the Chao-Gareyte scaling law which is given by

$$
\sigma_{l} \propto\left(\frac{\alpha I_{b}}{\nu_{s}^{2} E}\right)^{1 /(2+a)}
$$

where $a$ is the scaling factor. If we apply the Keil-Schnell criterion or $a=1$, we can obtain the bunch lengthening relation due to only the microwave instability as given by

$$
\sigma_{l}^{3}=\frac{\alpha c^{2} R\left|Z_{\|} / n\right|}{\sqrt{2 \pi}(E / e) \nu_{s}^{2} \omega_{o}^{2}} I_{b},
$$

where $\omega_{0}$ is revolution frequency.

The microwave instability also increases the energy spread when the beam current is higher than the threshold beam current. If we assume that the emittance is almost constant as the beam current is increased, the energy spread can be obtained from the horizontal beam size formula which is given by

$$
\sigma_{x}=\sqrt{\varepsilon_{x} \beta_{x}+\eta_{x}^{2} \delta^{2}},
$$

where $\sigma_{x}$ is the horizontal beam size, $\beta_{x}$ is the horizontal betatron function, $\eta_{x}$ is the horizontal dispersion function, and $\delta$ is the fractional energy spread.

\subsection{Combined Bunch Lengthening}

When the beam current is less than the threshold beam current, the bunch lengthening or shortening is generated only by the potential well distortion. However, when the beam current is higher than the threshold beam current, the bunch lengthening is generated by two combined actions of the microwave instability which also increases the energy spread and the potential well distortion which also generates the incoherent synchrotron frequency shift. In this case, total bunch lengthening due to the potential well distortion and the microwave instability is given by

$$
\sigma_{l}^{3}=\frac{\alpha c^{2} R}{\sqrt{2 \pi}(E / e) \nu_{s}^{2} \omega_{o}^{2}}\left\{\left|Z_{\|} / n\right|-\operatorname{Im}\left\{Z_{\|} / n\right\}_{\text {eff }}\right\} I_{b} .
$$

\section{TRANSVERSE EFFECTS}

\subsection{Head-Tail Instability}

When the chromaticity $\xi$ is nonzero, electrons at the head of a single bunch will affect the motions of the electrons at the tail of the bunch due to the finite resistivity of the surroundings. In this case, the transverse head-tail instability will be generated. The whole bunch oscillates transversely as a rigid unit for the azimuthal mode $m=0$ head-tail instability with the strongest growth rate than others. For $m= \pm 1$ mode head-tail instability, the head of the single

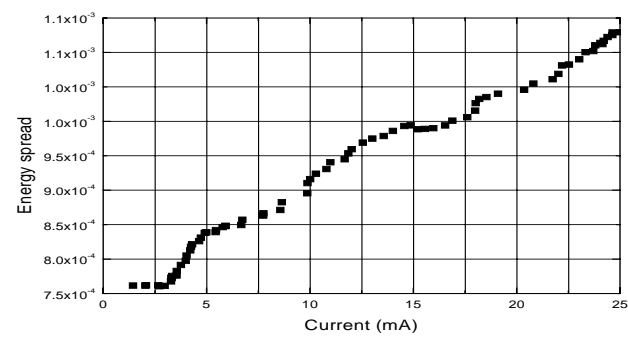

Figure 1: Energy spread with respect to single bunch beam current.

bunch oscillates transversely in one direction while the tail of the bunch oscillates transversely in the opposite direction with the stationary center of mass of the bunch. When the chromaticity $\xi$ is less than about 4 , the $m=0$ head-tail instability can be cured by putting the chromaticity nonzero positive. Since the growth rates of other mode head-tail instabilities are approximately proportional to the chromaticity, the other mode head-tail instabilities can be also controlled by selecting a small positive chromaticity.

\subsection{Strong Head-Tail Instability}

When the beam current is high, several satellites with distances about the synchrotron frequency will be appeared around the betatron fractional tunes according to the azimuthal modes. This is the head-tail instability. As the beam current increases, amplitudes of some azimuthal modes may be larger than those of the tunes and their frequencies will changed. At a threshold beam current, some azimuthal modes will be coupled and can not be distinguished. We call this phenomenon as the strong-head tail instability or the transverse mode coupling instability. The beams will be lost when the strong-head tail instability is generated, and the strong head-tail instability can be generated though the chromaticity is zero.

\section{RESULTS}

\subsection{Increase in Energy Spread}

In the PLS storage ring, there are an X-ray diagnostic beamline for the transverse beam structure study and a visible light diagnostic beamline for the longitudinal beam profile study. In the X-ray diagnostic beamline, X-ray illuminates a BGO crystal and a CCD camera captures the transverse beam image. The beam sizes are measured for the currents from $1 \mathrm{~mA}$ to $25 \mathrm{~mA}$ and their energy spreads with respect to the single bunch beam current are obtained by Eq. (5) as shown in Fig. 1. The energy spreads are almost constant when the single bunch beam current is less than a threshold beam current of $2.2 \mathrm{~mA}$. On the contrary, when the beam current is higher than the threshold beam current, the energy spreads are increased due to the microwave instability. 


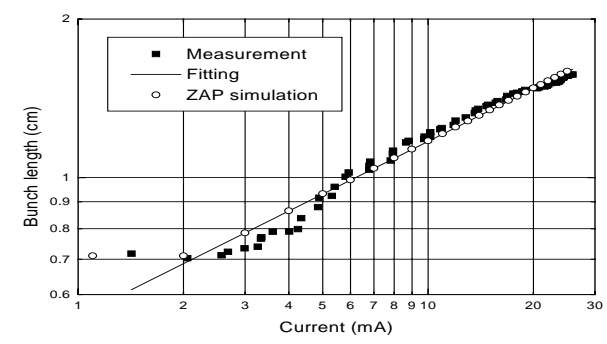

Figure 2: Single bunch length with respect to single bunch beam current: Measured bunch length with the fitting line, and calculated bunch length from the ZAP code simulation are plotted in log-log scale.

\subsection{Bunch Lengthening}

The single bunch lengths are measured by the Hamamatsu C5680 streak camera for the beam currents from $1 \mathrm{~mA}$ to $25 \mathrm{~mA}$ as shown in Fig. 2 where there are also ZAP code simulation results. The natural single bunch length is $0.72 \mathrm{~cm}$ and there is a threshold current about $2.2 \mathrm{~mA}$ which is the same as that of the energy spread. When the single bunch beam current is increased to the threshold, the bunch length is reduced a little due to the potential well distortion. However, its effect is ignorable as shown in Fig. 2.

On the contrary, when the beam current is increased from the threshold, the bunch length is increased according to a scaling law due to two combined actions of the microwave wave instability and the potential well distortion as shown in Fig. 2. In this case, the power of the single bunch length with respect to the single bunch beam current is about 0.33 which means the scaling factor $a$ of the Chao-Gareyte scaling law is about 1 by Eq. (3). The measured total longitudinal impedance due to the microwave wave instability and the potential well distortion is obtained as $0.56 \Omega$ from the fitting line of the measured bunch lengths $\sigma_{\tau}=0.55 \times I_{b}^{1 / 3}$ and Eq. (4).

According to the ZAP code simulation results, the effective longitudinal impedance $\operatorname{Im}\left\{Z_{\|} / n\right\}_{\text {eff }}$ due to the potential well distortion is about $-0.01 \Omega$, the calculated longitudinal broadband impedance $\left|Z_{\|} / n\right|$ due to the microwave instability is $0.54 \Omega$ which is much larger than the $\operatorname{Im}\left\{Z_{\|} / n\right\}_{\text {eff }}$, and the calculated transverse impedance $Z_{\perp}$ is $43.1 \mathrm{k} \Omega$. Therefore, the calculated total longitudinal impedance due to the microwave wave instability and the potential well distortion is about $0.54-(-0.01)=0.55 \Omega$ by Eq. (6), which is well agreed with the measured total longitudinal impedance of $0.56 \Omega$.

\subsection{Head-Tail and Strong Head-Tail}

The maximum single bunch beam current is about $30.17 \mathrm{~mA}$ when the horizontal and the vertical chromaticities are close to zero. However, the chromaticities are increased further by increasing the currents of the focusing and defocusing sextupole magnets by $5 \%$ and $10 \%$,

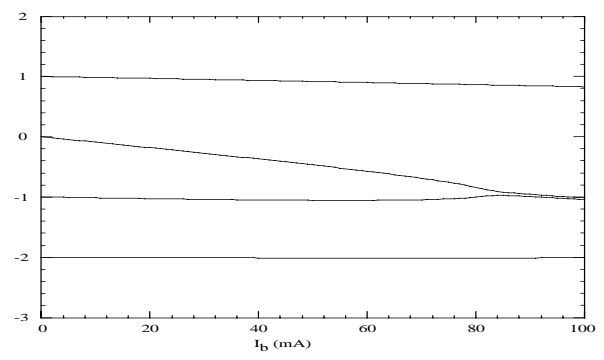

Figure 3: Strong head-tail simulation with MOSES code. Here, the horizontal and the vertical axes mean the single bunch beam current and the $\operatorname{Re}\left[\left(\nu-\nu_{x}\right) / \nu_{s}\right]$, respectively.

the maximum single bunch beam currents are decreased to $26.5 \mathrm{~mA}$ and $23.15 \mathrm{~mA}$, respectively. In these cases, there are the first synchrotron sidebands around the fractional betatron tunes. These are mainly due to the undamped $m= \pm 1$ mode head-tail instabilities even though the $m=0$ mode head-tail instability is damped down for the chromaticity range. Although we can cure those headtail instabilities by increasing the chromaticities much further, the beam lifetime can be also reduced due to the reduced dynamic aperture [4]. Therefore, the head-tail instabilities is controlled by changing the chromaticities. Up to the maximum single bunch beam current, we did not observe any mode coupling. Therefore, the threshold current of the head-tail instability is lower than that of the strong head-tail instability. From the MOSES code simulation, we have obtained a similar expectation of the threshold current for the strong head-tail instability as shown in Fig. 3. Here, there are the several azimuthal modes of $m=1,0,-1,-2$, and frequencies of $m=0$ and -1 modes become one at about $80 \mathrm{~mA}$ which is the threshold beam current of the strong head-tail instability. Therefore, we never observe any strong head-tail instability at near $30 \mathrm{~mA}$.

\section{SUMMARY}

In the PLS storage ring, single bunch collective effects are investigated. The threshold beam currents of the bunch lengthening and the energy spread are about $2.2 \mathrm{~mA}$, and the measured total longitudinal broadband impedance is about $0.56 \Omega$ which is well agreed with the ZAP code simulation. In the PLS storage ring, the most harmful transverse instability is the undamped $m= \pm 1$ mode head-tail instabilities which can be controlled by the chromaticities.

\section{REFERENCES}

[1] M. S. Zisman et al., "ZAP User's Manual,” LBL-21270, 1986.

[2] Y. H. Chin, "User's Guide for New MOSES Version 2.0," CERN LEP-TH/88-05, 1988.

[3] B. Zotter, "Potential-Well Bunch Lengthening," CERN SPS/81-14 (DI), 1981.

[4] M. P. Level et al., "Transverse Mode-Coupling Experiment in DCI," LAL/RT/84-09, 1984. 